\title{
Guidewire malposition during central venous catheterization despite the use of ultrasound guidance
}

\author{
Youn Joung $\mathrm{Cho}^{1}$, Sun Sook $\mathrm{Han}^{2}$, and Sang Chul Lee ${ }^{1}$ \\ Department of Anesthesiology and Pain Medicine, ${ }^{1}$ Seoul National University Hospital, Seoul National University College of \\ Medicine, ${ }^{2}$ Kangbuk Samsung Hospital, Sungkyunkwan University School of Medicine, Seoul, Korea
}

Central venous catheterization has become a mandatory part of clinical management in a variety of circumstances. It is a safe procedure in most patients, but it may be associated with a number of complications such as arterial puncture, hematoma, and malposition [1]. Ultrasound guidance for catheterization has become an accepted tool to improve the success rate and to reduce the number of attempts and complications. However, it does not prevent all complications. We encountered a case of the inadvertent insertion of a guidewire into the suspected subcutaneous area despite the use of ultrasound guidance.

A 77 -year-old woman (body mass index $15.9 \mathrm{~kg} / \mathrm{m}^{2}$ ) presented for vertebral corpectomy under suspicion of infectious spondylitis of the T12-L1 vertebrae. Her medical history included hypertension and previous operation for a compression fracture of the T12 and L1 vertebral bodies. Because the current operation needed to be performed in the prone position, anesthesia was induced on a stretcher, from which the patient would be moved to a prone position on the operating table. Monitoring with electrocardiography, non-invasive blood pressure, pulse oximetry, and bispectral index, anesthesia was induced and maintained by target-controlled propofol and remifentanil. Rocuronium was injected to facilitate tracheal intubation. After the muscles were relaxed, her right radial artery was cannulated for continuous invasive blood pressure monitoring, and the trachea was intubated and mechanical ventilation was started.

Central venous catheterization was planned for venous access and hemodynamic monitoring. The patient's head was turned slightly towards the left while preventing extreme rotation, which leads to overlapping of the internal jugular vein (IJV) and the common carotid artery. The patient's right anterior neck was prepped in a sterile manner. Next, the ultrasound probe was covered with a sterile sheath. The right IJV was assessed at the level of the cricoid cartilage using B-mode duplex sonography with a 5- to $13-\mathrm{MHz}$ linear transducer (LOGIQe; GE Medical, Jinagsu, China) using a standardized technique applying minimal probe pressure to obtain an adequate image. The size of the IJV looked rather small, and the patient seemed to be dehydrated or in a hypovolemic state. Intravenous fluid was administered, and positive end-expiratory pressure (PEEP) of $10 \mathrm{cmH}_{2} \mathrm{O}$ was applied to increase the size of the IJV. A shortaxis approach was used with dynamic ultrasound guidance during needle puncture. The operator controlled the needle with assistance by the attending anesthesiologist, who held the transducer with sterile sheath. The needle tip was visualized within the vessel, and dark venous blood regurgitation was confirmed through the syringe in a single attempt. Then, the ultrasound probe was taken off and the guidewire was introduced without significant resistance. However, subsequent ultrasonography using short- and long-axis views revealed that the guidewire was located in the surrounding subcutaneous tissue rather than within the vessel (Fig. 1A).

The guidewire was removed and then another needle puncture was attempted in which subsequent placement of a new guidewire was achieved uneventfully with ultrasonographic

Corresponding author: Sun Sook Han, M.D., Ph.D., Department of Anesthesiology and Pain Medicine, Kangbuk Samsung Hospital, Sungkyunkwan University School of Medicine, 108, Pyeong-dong, Jongno-gu, Seoul 110-746, Korea. Tel: 82-2-2001-1376, Fax: 82-2-2001-2313, E-mail: hss9438@empal.com

(c) This is an open-access article distributed under the terms of the Creative Commons Attribution Non-Commercial License (http:// creativecommons.org/licenses/by-nc/3.0/), which permits unrestricted non-commercial use, distribution, and reproduction in any medium, provided the original work is properly cited. 

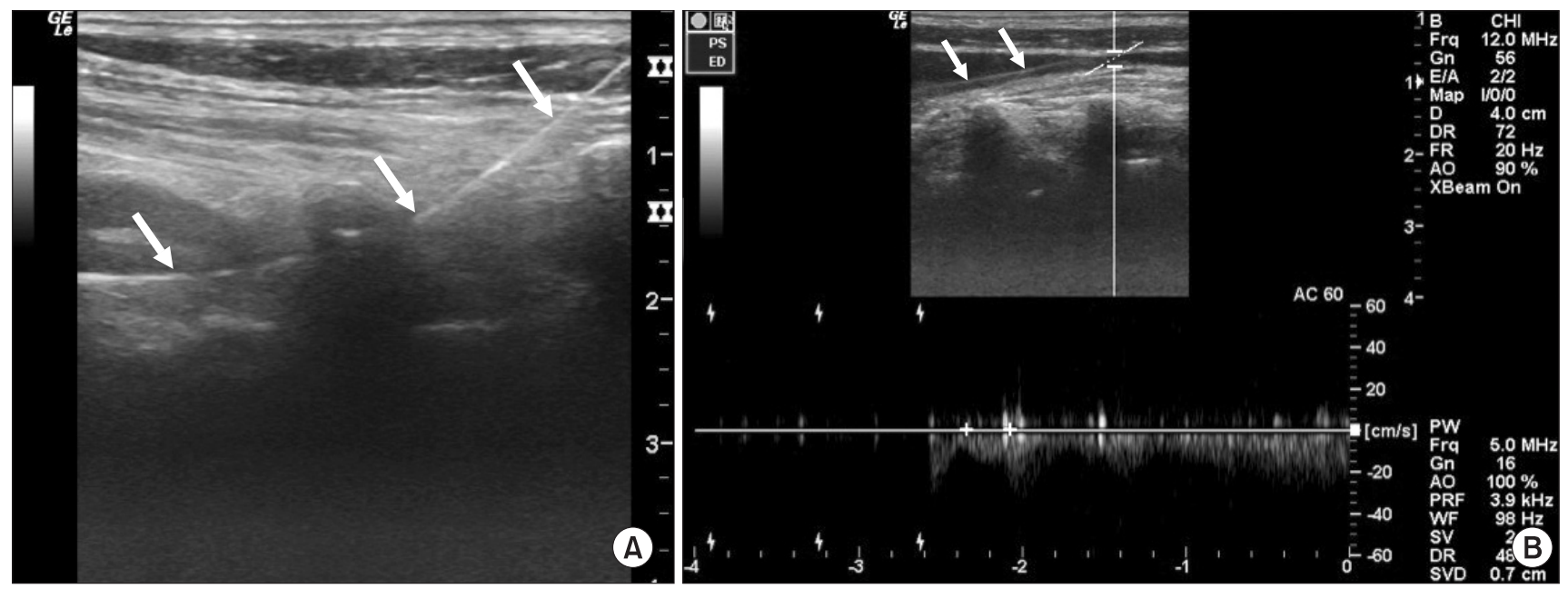

Fig. 1. Malposition of guidewire (arrows) in the suspected subcutaneous area (A). Pulse wave Doppler revealed that the guidewire (arrows) was located in the vein (B).

guidance. The intravascular location of the new guidewire was confirmed by rescanning the vein in both short- and longaxis views. Subsequent pulse-wave Doppler also revealed that the guidewire was located within the vein (Fig. 1B). After confirming the position of the guidewire, 7-Fr double-lumen central venous catheter (Arrow International, Inc., Reading, PA) was inserted over the wire and advanced into the IJV.

One explanation for the occurrence of the complications despite using ultrasound guidance is that the ultrasound beam is narrow, and although the operator appears to be following the tip of the needle in the vessel on the image, the real tip could actually have passed beyond the ultrasound beam and out through the contralateral wall of the vessel [2]. Furthermore, real-time imaging with ultrasound shows significant compression of the vein when a needle punctures the skin overlying the vein before entry into the lumen. The force applied to puncture the anterior wall of the vessel usually causes perforation of the posterior wall when the distance between the anterior and the posterior wall is small [3].

In our case, the patient's vein looked small in size. Thus, intravenous fluid was administered for volume support, and endexpiratory positive pressure of $10 \mathrm{cmH}_{2} \mathrm{O}$ was applied to increase the cross-sectional area of the IJV [4]. The Trendelenburg tilt position can also dilate the IJV by increasing the height between the right atrium and the right IJV, which decreases venous return to the heart via the superior vena cava, and hence potentially increases the chances of successful jugular cannulation [4]. Unfortunately, the stretcher where the anesthesia was conducted in our case was not able to take the Trendelenburg tilt position because of its equipment control limitation.

It is notable that incorrectly placing the needle outside of the targeted vessel can occur after passage through the vessel and concomitant blood withdrawal into the syringe [5]. The closer the needle tip is to the posterior wall, the more likely it is to migrate through the wall [5]. In our case, blood regurgitation was successful through the guide needle but it did not exclude malposition of the guidewire. We suspect that in the first attempt to insert the guidewire, the guidewire tip penetrated the collapsed fragile vein, and it then was misplaced in an extravascular location. In the subsequent trial, we checked proper guidewire position with a long-axis view and pulsewave Doppler. Checking the long-axis view and pulse-wave Doppler can be performed in mere seconds and would give great accuracy without requiring extra equipment. Although the initial introduction of the guidewire was uneventful, ultrasonography revealed unintended malposition of the guidewire, therefore preventing potential malposition of the catheter.

It is important to note that even with use of an ultrasoundguided procedure, an unrecognized complication can occur. In light of the current report, the clinician may wish to consider the need for a long-axis view and pulse-wave Doppler in addition to the usual short-axis method during central venous catheterization, even if the insertion proceeded smoothly and without apparent difficulties.

\section{References}

1. McGee DC, Gould MK. Preventing complications of central venous catheterization. N Engl J Med 2003; 348: 1123-33.

2. Levitov AB, Aziz S, Slonim AD. Before we go too far: ultrasoundguided central catheter placement. Crit Care Med 2009; 37: 2473-4.

3. Tercan F, Oguzkurt L, Ozkan U, Eker HE. Comparison of ultrasono- 
graphy-guided central venous catheterization between adult and pediatric populations. Cardiovasc Intervent Radiol 2008; 31: 575-80.

4. Marcus HE, Bonkat E, Dagtekin O, Schier R, Petzke F, Wippermann J, et al. The impact of Trendelenburg position and positive endexpiratory pressure on the internal jugular cross-sectional area.
Anesth Analg 2010; 111: 432-6.

5. Blaivas M, Adhikari S. An unseen danger: frequency of posterior vessel wall penetration by needles during attempts to place internal jugular vein central catheters using ultrasound guidance. Crit Care Med 2009; 37: 2345-9. 